\title{
Efecto de Fibrina Rica en Plaquetas en el postoperatorio de cirugía del tercer molar mandibular
}

\section{Effect of platelet-rich fibrin in the postoperative period of mandibular third molar surgery}

\section{Efeito da fibrina rica em plaquetas no período pós-operatório da cirurgia do terceiro molar mandibular}

doi) http://dx.doi.org/10.35954/SM2020.39.2.3

Walter Paz a (D) https://orcid.org/0000-0003-4794-2065

(a) Jefe de Servicio Odontológico. Regimiento "Gral. Aparicio Saravia" de Caballería Mecanizado № 7.

\section{RESUMEN}

Objetivos: Evaluar la fibrina rica en plaquetas como potenciador de la cicatrización y su posible efecto en la disminución de las morbilidades asociadas a la cirugía del tercer molar mandibular retenido y semiretenido. Reportar los factores causales de la retención del tercer molar mandibular y las posibles complicaciones asociadas a la extracción quirúrgica de dicha pieza.

Describir el principio y la función de los concentrados plaquetarios, sus aplicaciones clínicas y mecanismos de acción.

Proporcionar un examen completo de la literatura oral y maxilofacial publicada sobre fibrina rica en plaquetas, cómo evolucionó y se desarrolló, hasta donde está hoy; y cuáles son sus características biológicas, composición, protocolo de realización y cómo estos elementos clave funcionan dentro del entorno clínico.

Metodología: Para la obtención de datos del presente artículo se consultó la base Medline/PubMed y el portal Timbó Foco, sin aplicar límites de tiempo, en idioma inglés, español y portugués, además de textos utilizados en la carrera Doctor en Odontología de la Universidad de la República. Se revisaron estudios potencialmente relevantes que analizan el efecto de fibrina rica en plaquetas en el proceso de curación posterior a la cirugía del tercer molar mandibular retenido y semi-retenido y evalúan su posible efecto benéfico en las complicaciones postoperatorias asociadas a dicho procedimiento.

Conclusiones: Se confirma que fibrina rica en plaquetas disminuye, pero no previene algunas de las complicaciones postoperatorias asociadas a la cirugía del tercer molar mandibular logrando una mejoría significativa del dolor, edema y disminución en la incidencia de alveolitis. Fibrina rica en plaquetas parece acelerar la cicatrización en la cirugía del tercer molar mandibular induciendo la regeneración de tejidos blandos y duros. El procedimiento de preparación de fibrina rica en plaquetas es simple y rentable haciendo de su uso atractivo para muchos clínicos pudiendo ser aplicado en entornos clínicos ambulatorios.

PALABRAS CLAVE: Complicaciones Postoperatorias; Diente Impactado; Extracción Dental; Fibrina Rica en Plaquetas; Procedimientos Quirúrgicos Orales; Tercer Molar. 


\section{ABSTRACT}

Objectives: To evaluate platelet-rich fibrin as a healing enhancer and its possible effect on the reduction of morbidities associated with retained and semi-retained mandibular third molar surgery.

To report the causal factors of mandibular third molar retention and the possible complications associated with the surgical extraction of the mandibular third molar.

Describe the principle and function of platelet concentrates, their clinical applications and mechanisms of action.

To provide a comprehensive review of the published oral and maxillofacial literature on platelet-rich fibrin, how it evolved and developed to where it is today; and what are its biological characteristics, composition, performance protocol and how these key elements function within the clinical setting.

Methodology: To obtain data for this article we consulted the Medline/PubMed database and the Timbó Foco portal, without applying time limits, in English, Spanish and Portuguese, as well as texts used in the Doctor of Dentistry program at the Universidad de la República. Potentially relevant studies analyzing the effect of platelet-rich fibrin in the healing process after mandibular retained and semi-retained third molar surgery and evaluating its possible beneficial effect on postoperative complications associated with this procedure were reviewed.

Conclusions: It is confirmed that platelet-rich fibrin decreases but does not prevent some of the postoperative complications associated with mandibular third molar surgery achieving a significant improvement in pain, edema and decrease in the incidence of alveolitis. Platelet-rich fibrin appears to accelerate healing in mandibular third molar surgery by inducing soft and hard tissue regeneration. The platelet-rich fibrin preparation procedure is simple and cost-effective making its use attractive to many clinicians and can be applied in outpatient clinical settings.

KEY WORDS: Postoperative Complications; Tooth, Impacted; Tooth Extraction; Platelet-Rich Fibrin; Oral Surgical Procedures; Molar, Third.

\section{RESUMO}

Objetivos: Avaliar a fibrina rica em plaquetas como potencializador de cura e seu possível efeito na redução de morbidades associadas à cirurgia do terceiro molar mandibular retido e semi-retido.

Relatar os fatores causais da retenção do terceiro molar mandibular e as possíveis complicações associadas com a extração cirúrgica do terceiro molar mandibular.

Para descrever o princípio e a função dos concentrados plaquetários, suas aplicações clínicas e mecanismos de ação.

Fornecer uma revisão abrangente da literatura publicada sobre fibrina rica em plaquetas, como ela evoluiu e se desenvolveu até onde está hoje; e quais são suas características biológicas, composição, protocolo de desempenho e como esses elementos-chave funcionam dentro do cenário clínico.

Metodologia: A fim de obter dados para este artigo, o banco de dados Medline/Pubmed e o portal Timbó Foco foram consultados, sem aplicar limites de tempo, em inglês, espanhol e português, além de textos utilizados no curso de Doutorado em Odontologia da Universidad de la República. Estudos potencialmente relevantes analisando o efeito da fibrina rica em plaquetas no processo de cicatrização após a cirurgia mandibular retida e semi-retida de terceiros molares e avaliando seu possível efeito benéfico nas complicações pós-operatórias associadas a tal procedimento foram revisados. 
Conclusões: Está confirmado que a fibrina rica em plaquetas diminui, mas não evita algumas das complicações pós-operatórias associadas à cirurgia do terceiro molar mandibular, ao alcançar melhora significativa na dor, edema e diminuição da incidência de alveolite. A fibrina rica em plaquetas parece acelerar a cicatrização na cirurgia do terceiro molar mandibular, induzindo a regeneração dos tecidos moles e duros. O procedimento de preparação da fibrina rica em plaquetas é simples e econômico, tornando seu uso atraente para muitos clínicos e pode ser aplicado em ambiente clínico ambulatorial.

PALAVRAS ChAVE: Complicações Pós-Operatórias; Dente Impactado; Extração Dentária; Fibrina Rica em Plaquetas; Procedimentos Cirúrgicos Bucais; Dente Serotino.

\section{INTRODUCCIÓN}

La extracción quirúrgica del tercer molar $(3 \mathrm{M})$ mandibular retenido o semi-retenido, es uno de los procedimientos quirúrgicos más frecuentemente realizados en odontología. La prevalencia de complicaciones postoperatorias asociadas a este procedimiento es elevada por lo que encontrar una estrategia novedosa para reducir estas morbilidades resulta crucial. Aunque fibrina rica en plaquetas (PRF = platelet-rich fibrin) ha sido utilizado ampliamente en diferentes campos de la odontología para optimizar la cicatrización de heridas en los tejidos blandos y duros, la función precisa del PRF como potenciador de la cicatrización en la cirugía del 3M mandibular retenido o semi-retenido sigue siendo incierto. Las investigaciones plantean la hipótesis de que PRF podría ser beneficioso para reducir las morbilidades postoperatorias de la cirugía del 3M mandibular (1). Después de realizada la cirugía, en la fase postoperatoria temprana, los pacientes suelen presentar complicaciones como dolor, edema y trismo. Estas complicaciones inflamatorias son cruciales para los cirujanos para así desarrollar una estrategia personalizada de manera de reducir el riesgo de complicaciones y mejorar la curación postoperatoria. Varios intentos con Plasma rico en plaquetas $(\mathrm{PRP}=$ platelets-rich plasma), administración de antibióticos de manera pre y postoperatoria, crioterapia, drenaje de la herida, uso de diferentes tipos de colgajos, osteotomía con instrumentos de alta o baja velocidad de rotación, compresas de hielo en el postoperatorio, analgésicos, corti- coides y laser han sido realizados para reducir la evolución postoperatoria de la extracción del 3M mandibular post-cirugía (2).

PRF es una estrategia novedosa porque regula la inflamación y estimula el proceso inmunológico de quimiotaxis, perteneciendo a una nueva generación de concentrados plaquetarios con propiedades cicatriciales (3)

Con respecto a cirugía del 3M mandibular específicamente, algunos autores han descrito el beneficioso efecto de PRF en la reducción del dolor y osteítis alveolar después de la cirugía. Sin embargo, el verdadero efecto potencial de PRF sobre las complicaciones posquirúrgicas no está claro (1). El objetivo de esta revisión es responder la siguiente pregunta: ¿Hay alguna diferencia en las complicaciones postoperatorias (dolor, edema, trismo, alveolitis y cicatrización de tejidos duros y blandos) cuando se usa PRF en la cirugía del $3 \mathrm{M}$ retenido y semi-retenido? Otros resultados evaluados fueron el posible comportamiento ante la presencia de complicaciones hemorrágicas y alteraciones nerviosas.

\section{DESARROLLO}

La cirugía del 3M mandibular retenido o semierupcionado es uno de los procedimientos más comunes en odontología. Aun cuando es un procedimiento rutinario y usualmente electivo, el reporte de complicaciones es elevado. El desarrollo de los terceros molares mandibulares comienza en la rama mandibular aproximadamente a la edad de siete años. Son las piezas dentales que con más frecuencia se hallan retenidos y la pre- 
valencia de retención ha sido demostrada en un $56,5 \%$. El 3M en algunas ocasiones encuentra dificultades para corregir su dirección de erupción, debido a que su trayecto se encuentra debajo de la línea cervical del segundo molar. Esto provoca que el diente pueda encontrarse total o parcialmente retenido por tejido blando o duro (4-6). Los dientes retenidos rara vez eran un problema para el hombre neolítico. Su dieta altamente abrasiva causó el desgaste de los dientes, lo que resultó en una reducción de la distancia mesiodistal de la dentición. Esto permitió la migración mesial de los dientes y el espacio adecuado fue disponible para la erupción de los terceros molares. Pero con la llegada de alimentos refinados y la consecuente reducción en la carga funcional masticatoria, hoy, la tasa de retención de los terceros molares muestra un aumento significativo. Algunos factores que contribuyen a su retención son: formación tardía, variaciones raciales, aumento de densidad de hueso circundante, falta de espacio en la arcada debido a maxilares poco desarrollados, trastornos de tamaño y forma de los dientes, alteraciones genéticas, patrones de erupción erráticos, naturaleza de la dieta y grado de uso del aparato masticatorio (5).

Su extracción es indicada por propósitos preventivos y terapéuticos. Se indica su extracción para reducir el riesgo de secuelas, morbilidad y complicaciones que involucren a dientes vecinos y para mejorar la higiene oral. Las indicaciones incluyen prevención y tratamiento de pericoronaritis, caries no restaurables, patología pulpar y periapical, tratamiento del dolor idiopático, consideraciones ortodóncicas, diente debajo de prótesis, consideraciones en relación a las fracturas mandibulares, consideraciones periodontales y sistémicas, consideraciones sobre el segundo molar, prevención de quistes y tumores odontogénicos, consideraciones económicas, posición ectópica y como diente donante para trasplante. No existen dudas sobre su indicación de extracción cuando son la causa de dolor, infección o algún otro síntoma o signo. Por otra parte, la extracción qui- rúrgica de $3 \mathrm{M}$ mandibulares en pacientes asintomáticos sigue siendo un tema controversial $(6,7)$. La cirugía se asocia con frecuencia con dolor postoperatorio, edema y trismo, pudiendo tener un impacto tanto social como biológico, afectando la calidad de vida del paciente (8).

Bachmann et al en su estudio encontraron que la prevalencia de pacientes con complicaciones intra o postoperatorias producto de la cirugía de $3 \mathrm{M}$ fue de un $11 \%$. La complicación más frecuente fue la presencia de edema $(5,7 \%)$, seguido de dolor $(3,5 \%)$ y alveolitis $(2,4 \%)$. Otras complicaciones importantes pueden ocasionalmente incluir daño a la articulación temporo mandibular, disfunción transitoria del nervio alveolar inferior (NAI), disfunción permanente del NAI, infección, daño al diente adyacente y fractura mandibular (4).

En aquellos casos donde los dientes están profundamente impactados y cubiertos por una gran cantidad de hueso, la cirugía puede ser muy difícil, llevando a una mayor manipulación del tejido, un tiempo de operación más largo y consecuentemente más molestias postoperatorias $(1,9)$.

Por lo tanto, los clínicos tienen un gran interés en minimizar estas complicaciones, para mejorar la satisfacción del paciente y reducir el seguimiento adicional de visitas. La evidencia actual sobre estrategias postoperatorias para reducir estas complicaciones incluye crioterapia, medicamentos orales como analgésicos, corticoesteroides, antibióticos, así como también agentes tópicos como colutorios y geles antisépticos (8).

La regeneración exitosa y la curación pueden ser aceleradas por técnicas de regeneración ósea, por lo que varios estudios se han centrado en el desarrollo de tecnologías innovadoras para la regeneración tisular con buenas propiedades osteoinductiva y osteoconductiva. Biomateriales de fuentes autólogas, alogénicas y xenogénicas, materiales sintéticos, factores de crecimiento, citoquinas, o sus combinaciones son ampliamente utilizados, habiendo ventajas y desventajas asociadas con cada una de ellos. Las técnicas quirúrgicas realizadas para tener autoinjertos pueden 
ser difíciles y se asocian con una mayor morbilidad. Aun cuando las dificultades, se consideran el método patrón oro en la regeneración del tejido duro y blando, especialmente debido a su capacidad regenerativa autóloga. El desarrollo y la aplicación de los biomateriales han aumentado en los últimos años siendo las técnicas de ingeniería de tejidos muy importantes para la odontología y cirugía bucomaxilofacial (CBMF) (10).

El mayor desafío en los ensayos de investigación clínica es el desarrollo de materiales bioactivos para reducir los signos y síntomas postoperatorios mediante la regulación de la inflamación y aumentar la velocidad del proceso de curación $(11,12)$.

\section{Concentrados plaquetarios}

Un grupo interesante de biomateriales para aplicaciones locales son los productos derivados de la sangre. Varios tipos de biomateriales pueden ser purificados de la sangre humana, siendo conocidos generalmente como concentrados plaquetarios (13).

El uso de concentrados plaquetarios ha sido propuesto como una ayuda para mejorar la reparación de los tejidos. En cirugía oral pueden utilizarse como adyuvantes en la reparación de tejidos, durante las extracciones de dientes para estimular la curación, control de heridas y en prevención de sangrado postoperatorio en pacientes anti coagulados (14).

Recientemente han sido propuestos como una ayuda para mejorar regeneración de tejidos óseos y epiteliales en cirugía oral. Estudios in vitro, experimentos con animales y ensayos clínicos sugieren que los concentrados de plaquetas pueden desencadenar efectivamente la estimulación de la regeneración ósea y de tejidos blandos, reducir la inflamación, dolor y efectos secundarios no deseados (15).

En general, se clasifican en concentrados plaquetarios de primera y segunda generación dependiendo si existe o no adición de sustancias químicas adicionales agregadas con la finalidad de manipular el proceso de coagulación. Es así que tenemos productos concentrados con alta cantidad de plaquetas (PRP) como representantes de la primera generación y productos con alta cantidad de fibrina (PRF) representando a la segunda generación $(11,16)$.

Los concentrados plaquetarios han emergido como un potencial material regenerativo, utilizado de forma aislada o como andamiaje para otros materiales de injerto. El primer reporte data de 1970, siendo utilizado como "pegamento" para mejorar la cicatrización de heridas de piel. Tanto PRP como PRF usan sangre autóloga obtenida después de procesar una muestra de sangre completa, mediante centrifugación. Estos concentrados son innovaciones recientes que se utilizan en ingeniería de tejidos in vivo siendo suspensiones concentradas de factores de crecimiento en plaquetas que actúan como aditivos quirúrgicos bioactivos aplicados localmente para inducir la curación de heridas $(16,17)$.

\section{Plasma rico en plaquetas (PRP)}

PRP es un concentrado de plaquetas de primera generación. Se define como una concentración de plaquetas obtenida de sangre autóloga a través de centrifugación en un pequeño volumen de plasma y se considera una rica fuente de factores de crecimiento. Además, es rico en proteínas que actúan a nivel de la adhesión celular para la osteoconducción (fibrina, fibronectina y vitronectina), por lo que proporciona el soporte estructural necesario para la migración celular, y para la proliferación y crecimiento tridimensional de los tejidos sobre los que actúa $(13,18)$.

El potencial regenerativo de las plaquetas se debe a que las mismas contienen varios factores de crecimiento responsables del aumento de la mitosis celular, aumento de la producción de colágeno, crecimiento de los vasos sanguíneos, reclutamiento de células y muchos otros factores importantes en la curación o regeneración del tejido. Además, PRP actúa como un reservorio prometedor para factores de crecimiento que podría facilitar la cicatrización de heridas y la regeneración ósea $(17,19)$. 
Se introdujo por primera vez en 1998 por Marx, en combinación con injertos óseos autógenos para la reconstrucción de defectos mandibulares. En el campo de la cirugía oral se ha utilizado en diversos procedimientos clínicos con resultados variados dependiendo del tipo de preparación, como ser elevación del piso de seno maxilar, aumento de cresta alveolar, reconstrucción mandibular, reparación de fisuras en maxilar, tratamiento de defectos periodontales y en tratamientos de alveolos post extracción dental. Se ha aplicado solo o agregado a hueso autógeno, hueso anorgánico y sustitutos óseos minerales y orgánicos (18).

PRP podría aplicarse sobre la superficie del implante dental para aumentar el proceso de osteointegración y la formación de hueso nuevo después de la colocación del implante. Estudios muestran exitosos resultados después de la aplicación de PRP en el tratamiento de osteonecrosis de la mandíbula relacionada con bifosfonato (13). La desventaja de la preparación de PRP: que es una técnica sensible, compleja, costosa, y requiere mucho tiempo (al menos 30 minutos) hace que su uso sea poco práctico en muchos entornos clínicos ambulatorios (12).

Además, requiere la adición de anticoagulantes durante su procesamiento. Se ha demostrado que el uso de trombina bovina (promueve la conversión de fibrinógeno a fibrina) para su preparación puede tener efectos tóxicos en las células del cuerpo humano (11).

El término PRP se usó para nombrar todo tipo de técnicas y preparaciones. Estas se presentan sin una caracterización adecuada del contenido y arquitectura de los concentrados, por lo que conducen a datos contradictorios y controvertidos. Esto resultó en un estado de confusión que llevó a la sensación general de que los PRP no son tan útiles en el entorno clínico. En consecuencia, los PRP van lentamente desapareciendo debido a su complejidad de uso, costo de producción, restricciones legales en el manejo de procedimientos de sangre y resultados clínicos mixtos (19).

\section{Fibrina rica en plaquetas (PRF)}

En 2001, nació un concentrado de sangre de segunda generación, denominada fibrina rica en plaquetas (PRF) con numerosas ventajas y sin necesidad de anticoagulantes (10).

Fue desarrollado por primera vez en Francia por Choukroun para uso específico en cirugía oral y maxilofacial surgiendo como alternativa al PRP con numerosas ventajas tendiendo a convertirse en un adyuvante quirúrgico importante en procedimientos quirúrgicos orales. De hecho, solo después de la publicación de cinco importantes artículos en 2006 este biomaterial ganó atención en el campo de la odontología de investigación $(12,20)$. Es sabido que la matriz de fibrina es el producto final de la cascada de la coagulación siendo la forma activada de una molécula plasmática llamada fibrinógeno. Esta molécula fibrilar soluble esta masivamente presente tanto en plasma como en los gránulos $\alpha$ - de las plaquetas y juega un papel determinante en la agregación plaquetaria durante la hemostasia. Se transforma en un tipo de pegamento biológico constituyendo así una pared protectora a lo largo de las brechas vasculares durante la coagulación. Al ser una proteína soluble, el fibrinógeno se transforma en insoluble por la acción de la trombina, siendo el gel de fibrina polimerizado la primera matriz cicatrizal del sitio de la herida $(13,21)$. EI PRF se fundamenta en tres parámetros clave. Primero, la presencia de plaquetas y sus factores de crecimiento activados que están sustancialmente integrados en una sola matriz de fibrina durante el natural proceso de polimerización. Segundo, la presencia de leucocitos y sus citosinas que contribuyen a la acción anti infecciosa y regulación inmune en el proceso reparativo. Tercero, la densidad y la compleja organización de la arquitectura de la matriz de fibrina producida por una polimerización natural, sin la adición de ningún anticoagulante o gelificante. La fuerte arquitectura de la fibrina lo distingue de otros tipos de concentrados plaquetarios siendo un importante depósito de numerosos factores de crecimiento 
para promover la angiogénesis. Esta matriz de fibrina permite una lenta liberación de factores de crecimiento en la etapa de curación de la herida en un período de 7 a 14 días. Está compuesto de fibras delgadas con microporos que pueden servir como un andamio para la migración celular y diferenciación (20).

El estándar de oro para la curación y regeneración de tejidos in vivo requiere la interacción mutua entre un andamio (matriz de fibrina), plaquetas, factores de crecimiento, leucocitos y células madre. Estos elementos clave son todos los componentes activos del PRF, y cuando se combinan y preparan participan adecuadamente en los procesos de curación y regeneración de tejidos, incluida la proliferación y diferenciación celular, síntesis extracelular de la matriz, quimiotaxis y angiogénesis (neovascularización). Estudios clínicos revelan que este biomaterial es una matriz favorable para el desarrollo de una curación coherente, sin ningún exceso inflamatorio (19).

Recientemente, se ha informado que PRF podría estimular la proliferación de osteoblastos, fibroblastos gingivales, células pulpares, y células del ligamento periodontal, por otro lado, suprimen el crecimiento celular del epitelio oral. Estas acciones celulares específicas del PRF pueden ser beneficiosas para la regeneración de tejidos (22).

Beneficios de trabajar con PRF: facilidad de su técnica por lo que lleva menos tiempo, es económico para uso frecuente en la práctica asistencial am- bulatoria y para los pacientes ha demostrado tener un trauma mínimo. Además, la técnica es simple y no requiere anticoagulante ni ningún otro agente químico gelificante, no es nada más que sangre centrifugada sin ninguna adición $(11,17,21)$.

Se han reportado diferentes protocolos de centrifugación en la literatura, principalmente enfocados en modificar el tiempo (de 8 a 12 minutos) y la velocidad (de 2500 a 3200 rpm) lo que genera diferentes resultados en los concentrados y por ende resultados clínicos variables, posiblemente asociados a los cambios estructurales (23).

El protocolo de preparación de PRF es muy simple, una muestra de sangre es tomada sin anticoagulante en tubos que son inmediatamente centrifugados a $3000 \mathrm{rpm}$ (aproximadamente $400 \mathrm{~g}$ ) durante 10 minutos. La ausencia de anticoagulante implica la activación en unos minutos de la mayoría de las plaquetas de la muestra de sangre en contacto con las paredes del tubo, liberándose así la cascada de la coagulación. La técnica de obtención de PRF se fundamenta en la migración de los componentes sanguíneos por fuerza gravitacional, es así que el fibrinógeno inicialmente concentrado en la parte alta del tubo se combina con la trombina circulante debido a la centrifugación, para formar fibrina. El concentrado de sangre que se obtiene después la centrifugación tiene 3 capas distintas: un concentrado de glóbulos rojos en la parte inferior, un coágulo de fibrina (PRF) en el medio y una capa acelular sobrenadante de plasma (plasma pobre en plaquetas) en la parte superior $(12,19,21)$ (figura 1$)$.


Figura 1. A) Mesa auxiliar preparada con kit de recolección de sangre; B) Mesa auxiliar con centrífuga de mesa; C) Sangre inmediatamente centrifugada después de la recolección. 
A simple vista el coágulo de PRF se compone de dos partes principales: una porción amarilla de fibrina, constituyendo el cuerpo principal, y una porción roja llena de glóbulos rojos ubicada en el fondo del tubo. Entre estas dos áreas, puede ser observada una capa blanquecina llamada "capa leucocitaria" $(19,21)$ (figura 2).

Esta separación es estable y puede permanecer en el tubo por hasta 1 hora (24).

El coágulo de fibrina obtenido contiene aproximadamente un $95 \%$ de las plaquetas y un $50 \%$ de los leucocitos contenidos en la muestra original (16). Al momento de su utilización, se elimina la porción superior (sobrenadante de plasma), se recoge la fracción media separándola de la porción roja $2 \mathrm{~mm}$ debajo de la línea divisoria inferior. Las plaquetas están teóricamente atrapadas masivamente en la malla de fibrina (12).

PRF se puede usar directamente como un coágulo o después de la compresión, como membrana o tapón. Los clínicos pueden obtener una membrana de fibrina autóloga muy resistente al drenar los fluidos atrapados en la matriz de fibrina. Este procedimiento se puede realizar comprimiendo el biomaterial entre 2 gasas estériles. Se considera que de esta manera la cantidad de factores de crecimiento liberados de la membrana de PRF es menor, ya que el número de plaquetas dañadas es elevado debido a la compresión con gasa $(11,13)$. Una herramienta especial: la "caja metálica PRF" ha sido diseñada para recolectar y transformar hasta 16 coágulos de PRF en membranas con un espesor homogéneo de $1 \mathrm{~mm}$, en condiciones estériles y conservadas en un ambiente limpio y húmedo. Esta caja también contiene pozos de compresión y mazas para comprimir el PRF y transformarlo en cilindros densos $(11,17)$ (figura 3). Por lo tanto, si el PRF está comprimido a un estado de membrana, las células contenidas allí no se ven afectadas, de esta manera las plaquetas mantienen su integridad. Se incorporan $\geq 97 \%$ de plaquetas y aproximadamente la mitad de los leucocitos dentro de la membrana; y entre los leucocitos, los linfocitos se absorben específicamente.

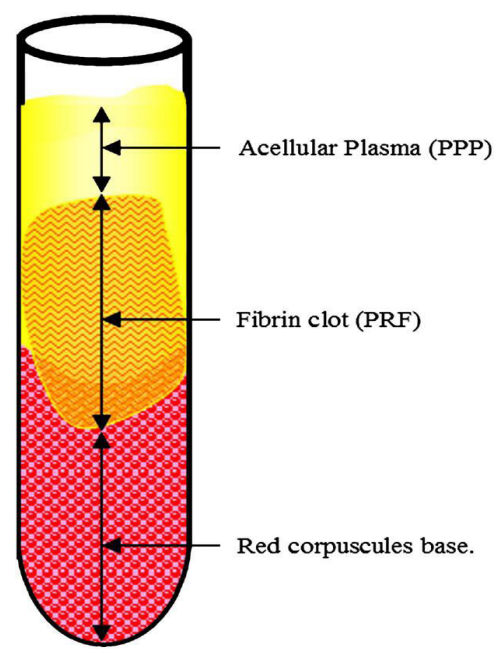

Figura 2. Esquema: coagulo de PRF en el medio, concentrado de glóbulos rojos en la parte inferior y plasma acelular en la parte superior. Tomado de Dohan et al. (21).

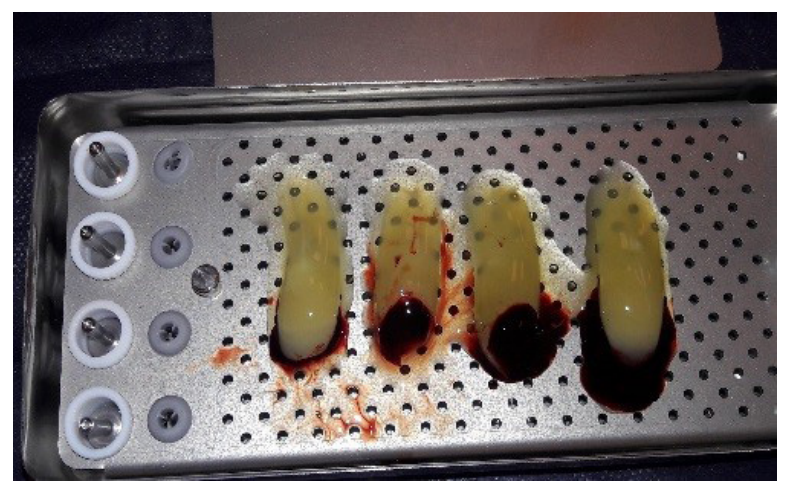

Figura 3. Caja conteniendo coágulos de PRF.

Histológicamente la membrana de fibrina es más densa que el PRF sin compactar, lo que comprueba la calidad del material para usarlo clínicamente como barrera $(23,25)$.

Estudios In vitro demuestran que esta membrana es capaz de liberar una cantidad significativa de factores de crecimiento autólogos (particularmente PDGF-AB, TGF $\beta$ y VEGF), citosinas y proteínas curativas (fibronectina, etc.) durante más de 7 días. La membrana tridimensional de fibrina es capaz de imitar la matriz extracelular en términos de su estructura, y crea el entorno o andamio para que las células funcionen óptimamente durante la curación y la regeneración $(13,14,19)$.

En los casos clínicos que se requiere usar el PRF como una matriz o andamio celular para la regeneración de tejidos como por ejemplo en lesiones de moderadas a severas, lo ideal es tener una red 
laxa que permita la migración celular, con espacios adecuados al tamaño de las células que se esperan alojar. Posiblemente este evento celular de migración podría ser más fácil en el PRF sin compactar que tiene espacios interfibrilares más amplios en comparación a la fibrina compactada. También se puede utilizar el PRF de forma combinada, en gel para mantener una estructura y proveer espacio para la regeneración tisular y además en membrana para posteriormente recubrir la lesión, mantener los tejidos y las estructuras de soporte. Alternativamente, el sobrenadante se puede aspirar del tubo y usarse en forma inyectable $(19,23)$. El éxito de esta técnica depende completamente de la velocidad de recolección de la sangre y transferencia a la centrífuga para su centrifugado dentro de los $2 \min 30 \mathrm{~s}$. El manejo rápido es la única forma de obtener un coágulo de PRF clínicamente utilizable, de hecho, sin anticoagulante, las muestras de sangre comienzan a coagular casi inmediatamente al contacto con el tubo de vidrio. Si la duración requerida para recolectar sangre y lanzar la centrifugación es demasiado largo ocurrirá una falla, la fibrina se polimerizará de manera difusa en el tubo y solo se obtendrá un pequeño coágulo de sangre sin consistencia. En conclusión, el protocolo PRF hace posible recoger un coágulo o gel de fibrina cargado con suero y plaquetas $(11,13,21)$.

En odontología PRF se ha utilizado ampliamente, solo o en combinación con otros materiales. PRF en forma de gel o coágulo se puede usar junto con injertos óseos, teniendo varias ventajas, como promover la cicatrización de heridas, el crecimiento óseo y maduración, sellado de heridas y hemostasia, e imparte mejores propiedades de manejo para injertar materiales (12).

Puede usarse como una membrana reabsorbible para la regeneración guiada del tejido evitando así la migración de células no deseables (células epiteliales) en el defecto óseo, proporcionando un espacio para la entrada de células osteogénicas y angiogénicas, y permitiendo la mineralización del coágulo sanguíneo subyacente. Otra diferencia entre PRF y un coágulo de sangre natural es que el PRF es más homogéneo, estable y más fácil de manejar y colocar localmente (17).

En el campo de la cirugía oral y bucomaxilofacial (BMF) ha sido evaluado en tratamientos quirúrgicos de defectos óseos, elevación de seno maxilar, implantología, preservación de alvéolos post extracción, cirugía periodontal, regeneración ósea, tratamiento quirúrgico en osteonecrosis de mandíbula relacionada con bifosfonato y cirugía de $3 \mathrm{M}$. PRF es ampliamente utilizado para la cirugía del tercer molar mandibular; sin embargo, su efecto sobre su potencial y beneficio sobre las complicaciones posquirúrgicas no están claras $(1,10,17)$. En cirugía oral y BMF se conocen dos tipos de cicatrización, por primera y segunda intención. La primera se logra con el uso de suturas, la segunda se basa en un cierre espontáneo de la herida. La cicatrización de un alvéolo postextracción sin la aplicación de materiales de relleno, se da por segunda intención. Esta cicatrización está producida en tres fases, la primera inflamatoria, siguiendo con la fase proliferativa y, por último, con la fase de remodelado (26).

Después de la extracción de un diente, un coágulo de sangre se forma en el alvéolo y el proceso de curación comienza. Los primeros eventos de curación incluyen la formación de tejido de granulación dentro del alvéolo, que es progresivamente reemplazado con hueso recién formado. El proceso de curación se considera completo después de 4 a 6 meses, una vez que la remodelación ósea ha tenido lugar (15).

La respuesta ideal de cualquier tejido ante una lesión que provoque perdida de su integridad es la formación de un coágulo que madure conforme transcurren las fases del proceso inflamatorio y concluya con la regeneración íntegra del tejido dañado. En los procesos de injuria sobre el tejido óseo se produce una interrupción vascular que crea un gradiente hipóxico en la zona de lesión, en donde la tensión de oxígeno en el centro de la 
herida es más baja. In vivo, este microambiente hipóxico estimula la expresión de una variedad de moléculas sintetizadas por las células inflamatorias, los fibroblastos, las células endoteliales y los osteoblastos, jugando un rol preponderante el Factor Inducible por Hipoxia (HIF-1 ${ }^{\mathrm{a}}$ ), complejo proteico constitutivo que regula la normoxia para cada tejido y también regula la transcripción de múltiples genes como ser PDGF, eritropoyetina, transferrina y VEGF. Este último, clave en la angiogénesis y recordemos es uno de las principales moléculas liberadas por el coágulo de PRF (16). La membrana de PRF tiende a iniciar la formación de nuevos vasos sanguíneos y epitelización facilitando una cobertura rápida de la herida con reabsorción ósea mínima en ciertas áreas (11). Para lo anterior es vital que las células endoteliales vecinas pueblen rápidamente el coágulo original en cantidad suficiente para asegurar un óptimo aporte de oxígeno, nutrientes y factores de crecimiento para el reclutamiento o diferenciación a las líneas celulares del tejido original, fibroblastos u osteoblastos (16).

Estudios in vitro demuestran que PRF es capaz de estimular la proliferación de osteoblastos, células del ligamento periodontal y fibroblastos gingivales durante un período de cultivo de 3 días, pero suprime el crecimiento de células epiteliales orales. La eficiencia de la aplicación local de PRF para controlar las complicaciones postoperatorias después de la extracción de un $3 \mathrm{M}$ retenido ha sido investigada por varios estudios $(2,13)$. Una complicación puede definirse como un "evento desagradable "que ocurre durante o después de una intervención quirúrgica. Las complicaciones durante la extracción del tercer molar son, básicamente, las mismas que las que pueden acontecer extrayendo cualquier otro diente; pero por la complejidad de la técnica de extracción de un diente retenido, y por las relaciones anatómicas propias del 3M son más frecuentes y tendrán unas características peculiares. Género, tipo y profundidad de impacto, nivel de dificultad, experiencia del cirujano, condición médica del paciente, así como fumar y usar anticonceptivos orales puede afectar las complicaciones postoperatorias $(27,28)$.

Hay datos muy limitados en la literatura sobre el efecto del PRF sobre el dolor, el trismo y edema en la cirugía del $3 \mathrm{M}$.

Los concentrados plaquetarios son un suministro efectivo para mejorar la curación de tejidos duros y blandos, lo que resulta en reducciones de dolor, edema y trismo. Sin embargo, hay algunos resultados controvertidos en la literatura, y ha habido un bajo número de estudios sistemáticos realizados hasta la fecha (28).

El curso postoperatorio normal tras la cirugía del $3 \mathrm{M}$ retenido y semi-retenido se caracteriza por ser relativamente molesto y por presentar en mayor o menor medida dolor. El dolor más fuerte se produce durante las primeras 24-72 horas, y decrece paulatinamente en los días sucesivos, pudiéndose prolongar durante una semana o más (5 a 10 días) (27).

Como ya se mencionó, el dolor puede ser considerado una complicación propia y esperable de un procedimiento quirúrgico invasivo. Estudios recientemente publicados no encontraron diferencias significativas en el valor promedio utilizando la Escala Analógica Visual (visual analog scale = VAS) entre el grupo PRF y el grupo control el día de la extracción del diente y el día 2 después de la cirugía, pero en los días 3 y 4 después de la cirugía, el grupo PRF mostró un valor VAS significativamente más bajo que el grupo control $(2,25)$. Luego de una revisión sistemática Canellas et al encontraron tres estudios con una diferencia estadísticamente significativa en dolor entre el PRF y el grupo control durante la primera semana de postoperatorio, solo un estudio no mostró una diferencia estadísticamente significativa entre los grupos para la evaluación del dolor (1).

Una razón sugerida para el efecto analgésico de PRF observado en el tercer y cuarto día después de la cirugía es el rápido progreso del tejido de curación. El hecho que PRF se puede transformar fácilmente en un estado de membrana después de ser sometido a compresión posibilitaría que algún 
efecto barrera proteja el sitio de la herida de la estimulación física en la cavidad oral. Después de la cirugía dental típica, el dolor se controla mediante la administración oral de varios tipos de analgésicos. Sin embargo, en embarazadas o mujeres lactantes o pacientes con alergia a medicamentos o trastorno hepático o renal, los analgésicos después de la extracción dental pueden ser riesgosos de administrar. Para tales pacientes, administrar PRF en el alvéolo extraído puede ser una gran ayuda para controlar el dolor, permitiendo una nueva utilidad en el campo de la cirugía oral y BMF (25). Un mayor despegamiento mucoperióstico, una ostectomía muy amplia, una técnica quirúrgica no reglada y traumática, un tratamiento previo insuficiente, actuaciones en fase aguda, etc. darán como resultado un postoperatorio más tormentoso. Por lo general, se produce un grado variable de inflamación (edema) de la zona maseterina y submaxilar. Esta inflamación aumenta durante las primeras 24-72 horas del postoperatorio dificultando la deglución y la apertura bucal (27). Esta evaluación se realiza mediante una guía horizontal y vertical utilizando una regla flexible y puntos visuales marcados en la cara. Para la guía horizontal, se mide desde el tragus de la oreja hasta el pogonion en tejido blando, que es el punto más prominente de la línea media de la barbilla. Para la guía vertical se mide desde el canto lateral del ojo al gonion (3).

Estudios mencionan resultados significativamente menores de inflamación y reducción del edema en los casos que se colocó PRF en comparación al grupo control fundamentalmente en los primeros días postoperatorios. En el séptimo día de postoperatorio las dimensiones de inflamación/edema fueron comparables sin diferencia significativa en ambos grupos (1-3).

Los estudios sobre PRF revelan que junto con la gran cantidad de factores de crecimiento liberados con la activación de plaquetas, también hay citoquinas inmunes incluidas como interleucina (IL) 1 beta, IL6, IL4 y factor de necrosis tumoral
(TNF) siendo este un motivo válido para su aplicación local en caso de inflamación (11).

La mayoría de los pacientes presenta en el postoperatorio una incapacidad o limitación para abrir la boca hasta los límites normales (trismus). El trismus es consecuencia de la irritación y el espasmo de los músculos masticadores especialmente el masetero por la inflamación y el trauma quirúrgico. Para evaluar el trismus, la abertura máxima de la boca se mide con pinzas manuales midiendo la distancia interincisiva. De acuerdo a varios estudios clínicos y de revisión no hubo diferencia estadísticamente significativa entre las puntuaciones de trismus en los casos donde se usó PRF y en el grupo control $(1,2,27)$.

La aplicación de PRF durante la extracción dental es capaz de acelerar la cicatrización de heridas, estimular la regeneración de tejidos óseos y blandos, y reducir los efectos secundarios no deseados (14).

Saluja et al en base a su experiencia clínica comprobó la potencia de PRF para estimular regeneración de tejidos blandos y duros después de la extracción dental encontrando que cavidades llenas de PRF mostraron una curación completa en la mitad del tiempo en comparación con el tiempo requerido para la curación fisiológica (12). La cicatrización de tejido blando se ve favorecida con el uso de este compuesto, los resultados mostraron una curación y cierre más rápido con diferencias estadísticamente significativas en los días 3 y 7 posteriores a la cirugía (3).

Para evaluar la curación ósea se utilizan diferentes métodos incluida evaluación radiográfica (densidad ósea, lámina dura y patrón trabecular) y medidas de gammagrafía (1).

Se muestran valores altamente significativos de densidad radiográfica ósea para el grupo PRF en el final del primer mes de seguimiento postoperatorio. Al seguimiento postoperatorio de 3 meses, hubo una significativa diferencia en la densidad ósea en el lado PRF en comparación con el lado de control en todos los pacientes (3). 
La curación de tejido óseo está dirigida por procesos tanto intracelulares como extracelulares. Las plaquetas cumplen un rol fundamental en la cicatrización de heridas formando coágulos de sangre y liberando factores de crecimiento. Su uso se basa en acelerar el proceso de cicatrización de tejidos blandos y tejido óseo (26).

En conclusión, las observaciones clínicas de PRF sugieren que la curación de tejidos incluye 4 aspectos: angiogénesis, control inmunitario, aprovechamiento de la circulación de células madre y protección de heridas por una cubierta epitelial (17). La alveolitis u osteítis alveolar es la complicación más común después de la cirugía del $3 \mathrm{M}$. Se caracteriza por dolor postoperatorio en y alrededor del sitio de extracción, que aumenta en severidad en cualquier momento entre 1 y 3 días después de la extracción, acompañado de una desintegración parcial o total del coágulo de sangre (fibrinólisis) dentro del alvéolo, pudiendo haber o no halitosis $(1,29)$.

La alveolitis es una complicación propia de este procedimiento, en su etiología multifactorial está involucrada la actividad bacteriana del Treponema dentícola, y su prevalencia se podría explicar por factores tales como la complejidad de la cirugía, la falta de irrigación durante el procedimiento operatorio, el cuidado postoperatorio por parte del paciente, la mala higiene bucal, pericoronaritis previa, el uso del tabaco o anticonceptivos orales $(4,27)$. Uno de los más importantes factores etiológicos de la osteítis alveolar es un aumento local de la actividad fibrinolítica y en consecuencia la degeneración del coágulo sanguíneo. Estudios mencionan un efecto beneficioso de PRF en comparación al coágulo natural encontrándose una diferencia significativa en la incidencia de osteítis alveolar. El coágulo de fibrina es muy fuerte y, en consecuencia, es menos susceptible a la acción fibrinolítica local y a la degradación del coágulo. Las propiedades inmunológicas del PRF, lo que resulta por su contenido en leucocitos, podría ser útil para prevenir las infecciones del sitio quirúrgico, con una consecuente reducción de los sín- tomas de inflamación, estimular la curación y el control de la herida. Estos conceptos pueden ayudar para explicar la baja prevalencia de osteítis alveolar con el uso de PRF en la extracción del $3 \mathrm{M}$ mandibular $(1,2)$.

Durante las primeras 12-24 horas es normal un ligero sangrado que rezuma del alvéolo, controlable mediante presión con una gasa. Sin embargo, a veces la pérdida de sangre es importante (27). Empero de los avances logrados en antihemorrágicos efectivos y técnicas quirúrgicas, encontrar agentes hemostáticos sigue siendo un problema persistente. Varios pacientes requieren habitualmente medicamentos anticoagulantes para prevención y tratamiento de complicaciones tromboembólicas asociado con condiciones médicas. Hay una gran variedad de agentes hemostáticos, como esponjas de colágeno, celulosa oxidada, antifibrinolíticos tópicos adhesivos sintéticos de cianoacrilato. Un estudio reciente propone a PRF como un agente hemostático para usar durante procedimientos quirúrgicos orales, encontrando que pacientes anti coagulados no mostraron signos de sangrado a las 24 horas después del procedimiento, que normalmente es observado en individuos que no reciben terapia con anticoagulantes. Además, la evaluación clínica inmediatamente después de la extracción dental mostró una disminución del sangrado que se mantuvo durante todo el proceso de curación. Se concluyó además que PRF podría ser utilizado de manera similar para el manejo del sangrado después de la extracción del tercer molar mandibular que está situado en un área a menudo propensa a varias complicaciones. El mecanismo de acción principal de este biomaterial posiblemente está relacionado a su capacidad de actuar como una barrera física funcional y facilita la activación de la cascada de coagulación que conduce al establecimiento de un coágulo estable en el sitio quirúrgico (30). Según la literatura, PRF es una herramienta útil en el control de la hemostasia posterior a la extracción, en la prevención de complicaciones hemorrágicas en pacientes cardiopáticos y con 
trastornos de la coagulación, en la profilaxis de hematomas postoperatorios, disminuir las tensiones del colgajo y acelerar el proceso de curación después de la cirugía $(13,14)$.

El nervio alveolar inferior (NAI) puede dañarse durante diversas intervenciones y la cirugía del tercer molar no escapa a esto. Estas lesiones pueden causar alteraciones transitorias o permanentes del nervio (27).

La deficiencia neuro sensorial resultante es una rara complicación $(0,4 \%$ y el $3 \%)$; sin embargo, debido a la frecuencia de este tratamiento, un número pequeño pero significativo de pacientes están afectados por este problema $(4,31)$.

Si la relación entre el $3 \mathrm{M}$ y el NAl es estrecha es posible que con nuestras maniobras puedan ser lesionados o traumatizados distintos fascículos del nervio. EI NAI se relaciona íntimamente con los ápices del tercer molar y durante la extracción de las raíces es relativamente fácil la presión sobre el conducto por donde discurre el nervio, la sección del nervio es mucho más rara, pero posible (26). Esta lesión puede manifestarse como hipoestesia, parestesia o disestesia resultando en un déficit neurosensorial muy significativo para los pacientes. Los efectos positivos de los factores de crecimiento en acelerar la curación y regeneración de los nervios se han informado recientemente en la literatura. Los vasos sanguíneos se pueden usar como un camino guía para dirigir la migración de las células de Schwann después de la lesión nerviosa periférica. La colocación de PRF en el lecho quirúrgico tendría efectos positivos en acelerar la curación y regeneración del NAI pudiendo servir como andamio rico en factores de crecimiento y mejorar la formación de vasos sanguíneos en la regeneración del tejido dañado. Por lo tanto, estos nuevos hallazgos sugieren que se puede lograr una mejor recuperación funcional para tratar la lesión clínica del NAI (31-33).

\section{CONCLUSIONES}

El desarrollo de la medicina regenerativa y la ingeniería tisular ha sido enorme en las últimas décadas, logrando posicionar técnicas y biomateriales en el quehacer cotidiano de diferentes especialidades de la odontología. PRF no es la excepción y aunque no se ha estudiado durante mucho tiempo, sí se puede utilizar de manera útil en cirugía oral y BMF para diversos procedimientos. Es biocompatible y puede inducir la regeneración de tejidos duros y blandos y lograr una respuesta postoperatoria exitosa para el paciente. La principal ventaja es que la preparación de PRF es sencilla, económica y utiliza la sangre del propio paciente reduciendo o eliminando la transmisión de enfermedades a través de la sangre. La extracción quirúrgica de terceros molares mandibulares ocasiona el trauma de los tejidos blandos y estructuras óseas en la cavidad bucal que pueden provocar un gran defecto óseo y en mayor o menor medida importantes complicaciones. El presente trabajo aborda este tema con una mirada más clínica y menos biológica para ayudar al lector a comprender con un poco más de profundidad su utilización en uno de los procedimientos más comunes en cirugía oral y BMF.

Existen datos muy limitados en la literatura sin embargo queda demostrado que PRF luego de la cirugía del 3M mandibular disminuye, pero no previene algunas de sus complicaciones postoperatorias logrando una mejoría significativa del dolor, edema y disminución en la incidencia de alveolitis. En resumen, el uso de PRF es un método alternativo para disminuir las complicaciones postoperatorias relacionadas a la cirugía del 3M mandibular. La realización de nuevos estudios clínicos es esencial para proporcionar evidencia más sólida para su aplicación clínica y establecerse como un tratamiento de buen pronóstico conduciendo a un mayor uso en la práctica odontológica ambulatoria.

DECLARACIÓN DE CONFLICTOS DE INTERESES: El autor no reporta ningún conflicto de interés. El estudio se realizó con recursos propios del autor y/o la institución a la que representa. 


\section{REFERENCIAS}

(1) Canellas JVDS, Ritto FG, Medeiros PJD. Evaluation of postoperative complications after mandibular third molar surgery with the use of plateletrich fibrin: a systematic review and meta-analysis. Int J Oral Maxillofac Surg 2017; 46(9):1138-1146. doi: 10.1016/j.ijom.2017.04.006

(2) Xiang X, Shi P, Zhang P, Shen J, Kang J. Impact of platelet-rich fibrin on mandibular third molar surgery recovery: a systematic review and meta-analysis.

BMC Oral Health [Internet].

2019 Jul 1 [cited 2020 Jun 13]; 19(1):1-10.

Available from: http://search.ebscohost.com.proxy. timbo.org.uy:2048/login.aspx?direct=true\&d$\mathrm{b}=$ edsdoj\&AN=edsdoj.8be1baaa9d94982bedda8f25b85f752\&lang=es\&site=eds-live

(3) Surekha K, Gudipalli S, Peramulla P, Prathima T, Kumar Y, Budumuru A. Efficacy of autologous platelet-rich fibrin in osseous regeneration after mandibular third molar surgery: A comparative study. Journal of Dr NTR University of Health Sciences [Internet]. 2019 Jan 1 [cited 2020 Aug 14]; 8(1):37-41.

Available from: http://search.ebscohost.com.proxy. timbo.org.uy:2048/login.aspx?direct=true $\& \mathrm{db}=\mathrm{ed}$ sdoj\&AN=edsdoj.313e118da4e45b850d8f9387 87759\&lang=es\&site=eds-live

(4) Bachmann H, Cáceres R, Muñoz C, Uribe S. Complicaciones en Cirugía de Terceros Molares entre los Años 2007-2010, en un Hospital Urbano, Chile. International journal of odontostomatology [Internet]. 2014 Apr 1 [citado 2020 Jul 4]; 8(1):107-12.

Disponible en: http://search.ebscohost.com.proxy. timbo.org.uy:2048/login.aspx?direct=true\&db=edssci\&AN=edssci.S0718.381X2014000100014\&lang= es\&site=eds-live

(5) Kumar Pillai A, Thomas S, Paul G, Singh SK, Moghe S. Incidence of impacted third molars: A radiographic study in People's Hospital, Bhopal, India. J Oral Biol Craniofac Res 2014; 4(2):76-81. doi: 10.1016/j.jobcr.2014.04.001
(6) Sánchez-Gutiérrez C, Cepeda-Bravo JA, Fierro-Serna VM, García-García Carlos E, Martínez-Rider R, Noyola-Frías MA. Eficacia del uso de Plasma rica en factores de crecimiento en defectos periodontales distales ensegundos molares inferiores, posterior a la extracción de un tercer molar mandibular.

Rev Esp Cirug Oral y Maxilofac [Internet]. 2017 Sep [citado 2020 June 12]; 39(3):164-170.

Disponible en: http://scielo.isciii.es/scielo.php?script =sci_arttext\&pid=S1130-05582017000300164\&lng=en. http://dx.doi.org/10.1016/j.maxilo.2016.04.001

(7) Crestanello Nese J. Aspectos controversiales en relación al manejo de los terceros molares asintomáticos. Actas Odontológicas 2010; 7(2):63-76.

(8) Cho H, Lynham AJ, Hsu E. Postoperative interventions to reduce inflammatory complications after third molar surgery: review of the current evidence. Australian dental journal [Internet]. 2017 Dec [cited 2020 Jul 4]; 62(4):412-9. Available from: http://search.ebscohost.com.proxy. timbo.org.uy:2048/login.aspx?direct=true \&db $=c m e d m \& A N=28498604 \&$ lang=es \&site=eds-live

(9) Rodríguez Aguirre GC, Martínez Herrera E, Duque Serna FL, Londoño Puerta LM.

Caracterización de terceros molares sometidos a exodoncia quirúrgica en la Facultad de Odontología de la Universidad de Antioquía entre 1991 y 2001. Rev Fac Odontol Univ Antioq [Internet]. 2009 Nov 6 [citado 2020 Jun 12]; 18(2). Disponible en: https://revistas.udea.edu.co/index. php/odont/article/view/2763

(10) Ghanaati S, Herrera-Vizcaino C, Al-Maawi S, Lorenz J, Miron RJ, Nelson K, et al. Fifteen Years of Platelet Rich Fibrin in Dentistry and Oromaxillofacial Surgery: How High is the Level of Scientific Evidence? The Journal of oral implantology [Internet]. 2018 Dec [cited 2020 Jun 13]; 44(6):471-92. Available from: http://search.ebscohost.com.proxy.timbo.org.uy:2048/login.aspx?direct=true $\& \mathrm{db}=$ cmedm\&AN=29870308\&lang=es\&site=eds-live 
(11) Raman S, Bhoj M, Madhulaxmi M, Jeevanandam G. Application of platelet-rich fibrin in oral and maxillofacial surgery - A short review. Drug Invention Today [Internet].

2019 Mar 15 [cited 2020 Jun 12]; 12(3):456-62. Available from: http://search.ebscohost.com.proxy. timbo.org.uy:2048/login.aspx?direct=true \&db=as$n \& A N=135900214 \&$ lang=es\&site=eds-live

(12) Saluja H, Dehane V, Mahindra U.

Platelet-Rich fibrin: A second generation platelet concentrate and a new friend of oral and maxillofacial surgeons. Annals of Maxillofacial Surgery [Internet]. 2011 Mar [cited 2020 Jun 15]; 1(1):53. Available from: http://search.ebscohost.com.proxy. timbo.org.uy:2048/login.aspx?direct=true \&db=edb\&AN $=64753592 \&$ lang $=e s \&$ site $=e d s-l i v e$

(13) Mihaylova Z, Mitev V, Stanimirov P, Isaeva A, Gateva N, Ishkitiev N. Use of platelet concentrates in oral and maxillofacial surgery: an overview. Acta odontológica Scandinávica [Internet]. 2017 Jan [cited 2020 Jun 13]; 75(1):1-11.

Available from: http://search.ebscohost.com.proxy. timbo.org.uy:2048/login.aspx?direct=true $\& d b=c-$ medm\&AN=27669885\&lang=es\&site=eds-live doi: 10.1080/00016357.2016.1236985

(14) Marenzi G, Di Lauro A, Sammartino G, Riccitiello F, Tia M. Influence of leukocyte- and platelet-rich fibrin (L-PRF) in the healing of simple postextraction sockets: A split-mouth study. BioMed Research International [Internet].

[Cited 2020 Jun 13]; 2015. Available from: http:// search.ebscohost.com.proxy.timbo.org.uy:2048/ login .aspx? direct $=$ true $\& d b=$ edselc $\& A N=e d$ selc. $2-52.0-84939208854 \&$ lang $=e s \&$ site $=e d s-l i v e$ doi: $10.1155 / 2015 / 369273$

(15) Del Fabbro M, Bortolin M, Taschieri S. Is autologous platelet concentrate beneficial for post-extraction socket healing? A systematic review. Int J Oral Maxillofacial Surg 2011; 40(9):891-900. doi: 10.1016/j.ijom.2011.04.009
(16) Correa AJ, Alister JP, Olate S, Manterola C. L-PRF y Ciclo Celular. Revisión narrativa. Int J Odontostomat 2019; 13(4):497-503.

(17) Feigin K, Shope B. Use of Platelet-Rich Plasma and Platelet-Rich Fibrin in Dentistry and Oral Surgery: Introduction and Review of the Literature. Journal of Veterinary Dentistry [Internet]. 2019 Jun [cited 2020 Jun 13]; 36(2):109.

Available from: http://search.ebscohost.com.proxy. timbo.org.uy:2048/login.aspx?direct=true\&db=edo\& $\mathrm{AN}=139414095 \&$ lang=es\&site=eds-live

(18) Plachokova AS, Nikolidakis D, Jansen JA, Mulder J, Creugers NHJ. Effect of platelet-rich plasma on bone regeneration in dentistry: Asystematic review. Clin Oral Impl Res 2008; 19(6):539-45. doi: 10.1111/j.1600-0501.2008.01525.x

(19) Hartshorne J, Gluckman H. A comprehensive clinical review of Platelet Rich Fibrin PRF) and its role in promoting tissue healing and regeneration in dentistry. Part 1: Definition, development, biological characteristics and function.

International Dentistry South Africa [Internet]. 2016 Oct [cited 2020 Jun 16]; 6(5):14.

Available from: http://search.ebscohost.com.proxy. timbo.org.uy:2048/login.aspx?direct=true \&db=edb\&AN=126887540\&lang=es\&site=eds-live

(20) Canellas JV d. S, Medeiros PJD, Figueredo CM d. S, Fischer RG, Ritto FG. Platelet-rich fibrin in oral surgical procedures: a systematic review and meta-analysis.

Int J Oral Maxillofacial Surg 2019; 48(3):395-414. doi: 10.1016/j.ijom.2018.07.007

(21) Dohan DM, Choukroun J, Diss A, Dohan SL, Dohan AJJ, Mouhyi J, et al. Platelet-rich fibrin (PRF): A second-generation platelet concentrate. Part I: Technological concepts and evolution. Oral Surgery, Oral Medicine, Oral Pathology, Oral Radiology and Endodontology [Internet]. 2006 Jan 1 [cited 2020 Jun 15]; 101(3):e37-44. doi: 10.1016/j.tripleo.2005.07.008 
(22) Assad M, Bitar W, Alhajj MN. Closure of Oroantral Communication Using Platelet-rich Fibrin: A Report of Two Cases. Annals of maxillofacial surgery [Internet].

2017 Jan [cited 2020 Jun 13]; 7(1):117-9.

Available from: http://search.ebscohost.com.proxy. timbo.org.uy:2048/login.aspx?direct=true\&db=cmedm\&AN=28713748\&lang=es\&site=eds-live

(23) Gutiérrez Ramirez DA, Hinojosa Sarria JP, Restrepo Dorado Al, Muñoz Ramírez AL,

Velarde trochez NF, Bastidas Daza FE.

Análisis estructural de la fibrina rica en plaquetas y sus aplicaciones en odontología regenerativa. Univ Odontol 2018; 37(79).

https://doi.org/10.11144/Javeriana.uo37-79.aefr

(24) Simon BI, Gupta P, Tajbakhsh S.

Quantitative evaluation of extraction socket healing following the use of autologous platelet-rich fibrin matrix in humans. International Journal of Periodontics and Restorative Dentistry [Internet]. [Cited 2020 Jun 14]; 31(3):285-95.

Available from: http://search.ebscohost.com.proxy. timbo.org.uy:2048/login.aspx?direct=true\&db=edselc\&AN=edselc.2-52.0-79960933109\&lang=es\&site=eds-live

(25) Miyamoto $\mathrm{H}$, Nakamura $\mathrm{T}$, Takashima $\mathrm{H}$, Mizutani T, Morita M, Hirose M, et al. Investigation of the analgesic effect of platelet-rich fibrin on postoperative pain after mandibular impacted wisdom tooth extraction. Journal of Oral and Maxillofacial Surgery, Medicine, and Pathology [Internet]. 2020 Jan 1 [cited 2020 Jul 3]; Available from: http://search.ebscohost.com.proxy.timbo.org. uy:2048/login.aspx?direct=true \&db=edselp\&AN= S2212555820300235\&lang=es\&site=eds-live doi: 10.1016/j.ajoms.2020.03.001
(26) Guzmán Castillo GF, Paltas Miranda ME, Benenaula Bojorque JA, Núñez Barragán Katerine I, Simbaña García DV. Cicatrización de tejido óseo y gingival en cirugías de terceros molares inferiores. Estudio comparativo entre el uso de fibrina rica en plaquetas versus cicatrización fisiológica. Rev. Odont. Mex [revista en la Internet]. 2017 Jun [citado 2020 Jun 23]; 21(2): 114-120. Disponible en: http://www.scielo.org.mx/scielo.php? script=sci_arttext\&pid=S1870-199X2017000200114\& Ing=es. http://dx.doi.org/10.1016/j.rodmex.2017.05.07

(27) Gay Escoda C, Piñera Penalva M, Valmaseda Castellón E. Cordales incluidos. Exodoncia quirúrgica. Complicaciones.

En: Cosme Gay Escoda, Leonardo Berini Aytes. Tratado de Cirugía Bucal. Tomo 1. pp. 436-37, 441-42, 454-56.

(28) Asutay F, Yolcu Ü, Geçör O, Acar AH, Öztürk SA, Malkoç $S$. An evaluation of effects of platelet-rich-fibrin on postoperative morbidities after lower third molar surgery.

Niger J Clin Pract 2017; 20(12):1531-1536.

doi: 10.4103/1119-3077.181400

(29) Daly B, Sharif MO, Newton T, Jones K, Worthington HV. Local interventions for the management of alveolar osteitis (dry socket) (Intervenciones locales para el tratamiento de la osteítis al veolar (alvéolo seco) Cochrane Database of Systematic Reviews 2012, Issue 12. Art. No.: CD006968. doi: 10.1002/14651858.CD006968.pub2

(30) de Almeida Barros Mourão CF, Alves GG, de Mello Machado RC, Calasans-Maia MD,

Miron RJ, Ghanaati S. Usefulness of platelet-rich fibrin as a hemostatic agent after dental extractions in patients receiving anticoagulant therapy with factor Xa inhibitors: a case series.

Oral and Maxillofacial Surgery 2019 [Internet].

[Cited 2020 Jun 23]; 23(3):381-6. Available from: http://proxy.timbo.org.uy/login?url=http://search. ebscohost.com/login.aspx?direct=true \&db=edsel$c \& A N=$ edselc. $2-52.0-85066025289 \&$ lang $=e s \& s i-$ te=eds-live 
(31) Blakey G. Extracciones de los terceros molars. En: Mehra P, D'Innocenzo R. Manual de Cirugía Oral Menor para el Odontólogo General. 2a. ed. Amolca, 2019. p. 80.

(32) Khojasteh A, Hosseinpour S, Nazeman P, Dehghan MM. The effect of a platelet-rich fibrin conduit on neurosensory recovery following inferior alveolar nerve lateralization: a preliminary clinical study. International Journal of Oral \& MaxiIlofacial Surgery [Internet].

2016 Oct 1 [cited 2020 Jun 14]; 45(10):13038.

doi: 10.1016/j.jom.2016.06.003

(33) Huang M-L, Zhai Z, Chen Z-X, Yang X-N, Qi Z-L. Platelet-rich fibrin membrane nerve guidance conduit: a potentially promising method for peripheral nerve injuries. Chinese medical journal [Internet]. [Cited 2020 Jul 3]; 133(8):999-1001.

Available from: http://search.ebscohost.com.proxy. timbo.org.uy:2048/login.aspx?direct=true\&d$\mathrm{b}=$ edselc\&AN=edselc.2-52.0-85083913781\&lan$\mathrm{g}=\mathrm{es} \&$ site=eds-live 